\title{
STIELTJES TRANSFORMS ON NEW GENERALIZED FUNCTIONS
}

\author{
JOHN SCHMEELK
}

(Received 27 December 1999)

\begin{abstract}
We introduce a Stieltjes transform on the equivalence classes of a new generalized function which has been successfully developed by Colombeau. Subsets of rapid descent test functions, $\mathscr{S}\left(\mathbb{R}^{n}\right)$, as well as tempered distributions, $\mathscr{S}^{\prime}\left(\mathbb{R}^{n}\right)$, are used to preserve Fourier analysis techniques.
\end{abstract}

2000 Mathematics Subject Classification. Primary 46F12.

1. Introduction. Schwartz was the recipient of the prestigious fields Medal in 1950 for his achievement in developing distributional theory. However he did indicate in his paper [5] the impossibility of having a distributional multiplication satisfying the associative, commutative as well as the multiplicative identity and multiplicative inverse axioms. Many users of generalized functions indicated partial solutions to many of these problems.

A recent and rigorous mathematical development preserving distributional multiplication has been successfully developed by Colombeau [1, 2, 3]. His new theory termed new generalized functions contain many new important discoveries. Integration techniques are also developed within the new generalized function framework. The approach is algebraic in nature whereby a new generalized function has an equivalence class of special functions representing it. It is within these equivalence classes where Colombeau introduces multiplication and integration. We will introduce a Stieltjes transform on these equivalence classes in much the same manner as Colombeau introduces the Fourier transform. Subsets of rapid descent test functions, $\mathscr{Y}\left(\mathbb{R}^{n}\right)$, as well as tempered distributions, $\mathscr{Y}^{\prime}\left(\mathbb{R}^{n}\right)$, will be used so as to preserve Fourier analysis techniques which are so well developed within these two spaces and required within the framework of applying Stieltjes transforms on new generalized functions.

2. New generalized functions. We begin with a brief review for the construction of new generalized functions. Colombeau reexamines the space of infinitely differentiable functions having compact support, $\mathscr{D}\left(\mathbb{R}^{n}\right)$, and defines a sequence of subspaces,

$$
\mathscr{A}_{q}=\left\{\phi \in \mathscr{D}\left(\mathbb{R}^{n}\right): \int_{\mathbb{R}^{n}} \phi(x) d x=1, \int_{\mathbb{R}^{n}} x^{\alpha} \phi(x) d x=0,1 \leq \alpha \leq q\right\}
$$

whereby

$$
\mathscr{D}\left(\mathbb{R}^{n}\right) \supset \mathscr{A}_{1}\left(\mathbb{R}^{n}\right) \supset \cdots \supset \mathscr{A}_{q}\left(\mathbb{R}^{n}\right) \supset \cdots,
$$

for $q=1,2, \ldots$, and $x^{\alpha}=x_{1}^{\alpha} x_{2}^{\alpha} \cdots x_{n}^{\alpha}$. 
It is clear that $\phi(x) \in \mathscr{A}_{\mathcal{q}}\left(\mathbb{R}^{n}\right)$ if and only if the function

$$
\phi_{\varepsilon}(x) \triangleq \frac{1}{\varepsilon^{n}} \phi\left(\frac{x}{\varepsilon}\right)
$$

is also a member of $\mathscr{A}_{q}\left(\mathbb{R}^{n}\right)$. We now define the set of moderate functions, $\varepsilon_{m}\left(\mathbb{R}^{n}\right)$.

DEFINITION 2.1.

$$
R \in \varepsilon_{m}\left(\mathbb{R}^{n}\right) \text { if and only if } R: \mathscr{A}_{1} \times\left(\mathbb{R}^{n}\right) \longmapsto \mathbb{C}, R:(\phi, x) \longmapsto R(\phi, x)
$$

are $C^{\infty}$ in $x$ for each $\phi \in \mathscr{A}_{1}\left(\mathbb{R}^{n}\right)$. Furthermore the function, $R$, must satisfy the condition that if for each compact subset, $K \subset \mathbb{R}^{n}$, and every partial derivative operator,

$$
D=\frac{\partial^{|k|}}{\partial x_{1}^{k_{1}} \cdots \partial x_{n}^{k_{n}}}
$$

then there is an $N \in \mathbb{N}$ (positive integers) such that for $\phi \in A_{N}, \exists \eta>0, C>0$ such that the inequality

$$
\left|D R\left(\phi_{\varepsilon}, x\right)\right| \leq \frac{C}{\varepsilon^{N}}
$$

is satisfied for every $x \in K$ and $0<\varepsilon<\eta$. Herein we establish the notation convention, $|k|=\sum_{i=1}^{n} k_{i}$, where the $k_{i}$ are positive integers.

Next the class of tempered moderate functions, $\varepsilon_{m, \tau}\left(\mathbb{R}^{n}\right)$ are defined whereby the requirement of a compact subset, $K \subset \mathbb{R}^{n}$, in Definition 2.1 is relaxed to be the entire space, $\mathbb{R}^{n}$, and moreover the inequality (2.6) is replaced with the inequality

$$
\left|D R\left(\phi_{\varepsilon}, x\right)\right| \leq \frac{C\left(1+|x|^{N}\right)}{\varepsilon^{N}}
$$

for all $x \in \mathbb{R}^{n}$ (see [2, 3]).

EXAMPLE 2.2. If $f(x) \in \mathscr{Y}\left(\mathbb{R}^{n}\right)$ the Schwartz space of classical rapid descent test functions, then the associated function

$$
R_{f}(\phi, x) \triangleq \int_{\mathbb{R}^{n}} f(\lambda) \phi(\lambda-x) d \lambda
$$

belongs to $\varepsilon_{m, \tau}\left(\mathbb{R}^{n}\right)$.

Proof. Recall that

$$
R_{f}\left(\phi_{\varepsilon}, x\right)=\int_{\mathbb{R}^{n}} \frac{1}{\varepsilon^{N}} f(\lambda) \phi\left(\frac{\lambda-x}{\varepsilon}\right) d \lambda,
$$

and denoting $o(D)$ to be the order of the partial differential operator, $D$, we have

$$
\begin{aligned}
\left(D R_{f}\left(\phi_{\varepsilon}, x\right)\right) & =\left(-\frac{1}{\varepsilon}\right)^{o(D)} \int_{\mathbb{R}^{n}} \frac{1}{\varepsilon^{N}} f(\lambda)(D \phi)\left(\frac{\lambda-x}{\varepsilon}\right) d \lambda \\
& =\left(-\frac{1}{\varepsilon}\right)^{o(D)} \int_{\mathbb{R}^{n}} f(x+\varepsilon \mu)(D \phi)(\mu) d \mu,
\end{aligned}
$$

and for fixed $\phi \in \mathscr{A}_{1}\left(\mathbb{R}^{n}\right)$ these integrations are in compact sets and thus we immediately have that $R \in \varepsilon_{m, \tau}\left(\mathbb{R}^{n}\right)$. 
NotATion CONVENTion. The set $\Gamma$ will be the collection of all increasing functions $\alpha$ on the set of positive integers $\mathbb{N}$ to the positive real numbers $\mathbb{R}^{+}$satisfying $\lim \alpha(q) \rightarrow \infty$ as $q \rightarrow \infty$.

DEFINITION 2.3. An ideal, $N_{\tau}\left(\mathbb{R}^{n}\right) \subset \varepsilon_{m, \tau}\left(\mathbb{R}^{n}\right)$ is as follows:

$$
\begin{aligned}
N_{\tau}\left(\mathbb{R}^{n}\right) \triangleq & \left\{R \in \varepsilon_{m, \tau}\left(\mathbb{R}^{n}\right) \text { whereby } \forall D, \exists N \in \mathbb{N},\right. \\
& \alpha \in \Gamma \text { such that if } q \geq N, \phi \in \mathscr{A}_{q} \\
& \exists C>0, \eta>0 \text { then } \\
& \left|D R\left(\phi_{\varepsilon}, x\right)\right| \leq C\left(1+|x|^{n}\right) \varepsilon^{\alpha(q)-N}, \\
& \left.\forall x \in\left(\mathbb{R}^{n}\right) \text { and } 0<\varepsilon<\eta\right\} .
\end{aligned}
$$

The space of new generalized functions is now defined in an algebraic sense where they are the collection of equivalence classes or coset classes as given by the quotient set,

$$
G_{\tau}\left(\mathbb{R}^{n}\right) \doteq \varepsilon_{m, \tau}\left(\mathbb{R}^{n}\right) /_{N_{\tau}\left(\mathbb{R}^{n}\right)} .
$$

That is to say two representatives, $R_{1}$ and $R_{2}$, belong to the same coset class if and only if $R_{1}\left(\phi_{\varepsilon}, x\right)-R_{2}\left(\phi_{\varepsilon}, x\right)$ belong to the ideal $N_{\tau}\left(\mathbb{R}^{n}\right)$.

3. Integration on new tempered generalized functions. The Stieltjes transform will require an integration process in a somewhat similar manner as does the Fourier transform. Since distributional integration eluded the mathematical community, we first give a brief review of new generalized function integration theory as developed by Colombeau [2, 3].

DefinITION 3.1. Let $G \in \mathscr{G}_{\tau}\left(\mathbb{R}^{n}\right)$ and $R \in \varepsilon_{m, \tau}\left(\mathbb{R}^{n}\right)$ be a representative for $G$. The integral, $I$, of $R$ is defined by the formula

$$
I_{R}\left(\phi_{\varepsilon}\right) \triangleq \int_{\mathbb{R}^{n}} R\left(\phi_{\varepsilon}, x\right)\left(\hat{\phi}_{\varepsilon}\right)(x) d x,
$$

where $\hat{\phi}_{\varepsilon}$ is the Fourier transform of $\phi \in \mathscr{A}_{N}$, where $0<\varepsilon<1$ (see [2, 3]).

Should $\phi \notin \mathscr{A}_{N}$ or if $\varepsilon>0$ is not sufficiently small, we set

$$
I_{R}\left(\phi_{\varepsilon}\right)=0
$$

We first observe that because $\phi_{\varepsilon}(x)=\left(1 / \varepsilon^{n}\right) \phi(x / \varepsilon)$, we immediately have

$$
\left(\hat{\phi}_{\varepsilon}\right)(w)=\int_{\mathbb{R}^{n}} e^{-i x w} \frac{1}{\varepsilon^{n}} \phi\left(\frac{x}{\varepsilon}\right) d x=\int_{\mathbb{R}^{n}} e^{-i \eta \varepsilon w} \phi(\eta) d \eta=\hat{\phi}(\varepsilon x),
$$

so that

$$
I_{R}\left(\phi_{\varepsilon}\right)=\int_{\mathbb{R}^{n}} R\left(\phi_{\varepsilon}, x\right)\left(\hat{\phi}_{\varepsilon}\right)(x) d x=\int_{\mathbb{R}^{n}} R\left(\phi_{\varepsilon}, x\right)(\hat{\phi})(\varepsilon x) d x .
$$

Now by the definition of $\varepsilon_{m, \tau}\left(\mathbb{R}^{n}\right)$, our representative $R$ satisfies

$$
\left|R\left(\phi_{\varepsilon}, x\right)\right| \leq C \frac{\left(1+|x|^{N}\right)}{\varepsilon^{N}}
$$


and moreover whenever $\phi \in \mathscr{A}_{N}$ we have $\hat{\phi} \in \mathscr{Y}\left(\mathbb{R}^{n}\right)$ so that

$$
|\hat{\phi}(x)| \leq \frac{C_{p}}{\left(1+|x|^{p}\right)}
$$

for all $x \in\left(\mathbb{R}^{n}\right)$. Putting $p=N+n+1$, we obtain

$$
\left|I_{R}\left(\phi_{\varepsilon}\right)\right| \leq \frac{C^{\prime}}{\varepsilon^{2 N+n+1}},
$$

(see [3]). Therefore, we can consider the integral $I_{R}\left(\phi_{\varepsilon}\right)$ as a function from $\mathcal{A}_{N} \times \mathbb{R}^{n} \mapsto \mathbb{R}$ satisfying the moderate estimate shown by inequality (3.7). Furthermore, using similar estimates on a representative belonging to the ideal, $N_{\tau}\left(\mathbb{R}^{n}\right)$, will also prove that its integral is again a member belonging to the ideal, $N_{\tau}\left(\mathbb{R}^{n}\right)$. Thus we conclude the integral of a new tempered generalized function does not depend upon the representative selected from its coset class.

We also note that the extra component, $\left(\hat{\phi}_{\varepsilon}\right)(x)$, used in the kernel of the integrand defined in expression (3.1) does not change the value of the integral. This is shown by directly comparing it to the value

$$
\int_{|x| \leq a} R\left(\phi_{\varepsilon}, x\right) d x
$$

This is clear by applying a Fourier transform on a $\phi \in \mathscr{A}_{q}$, where $\hat{\phi}(0)=1$ and $(D \hat{\phi})(0)=0$ for $1 \leq o(D) \leq q$. Using the Taylor formula on $\hat{\phi}$ one immediately has that

$$
|\hat{\phi}(\varepsilon x)-1| \leq C \varepsilon^{q+1}
$$

for $|x| \leq a$ and the difference between $\int_{|x| \leq a} R\left(\phi_{\varepsilon}, x\right) d x$ and $\int_{\mathbb{R}^{n}} R\left(\phi_{\varepsilon}, x\right)\left(\hat{\phi}_{\varepsilon}\right)(x) d x$ can be made as small as we please (see [3]).

4. Fourier transforms on new generalized functions. The Stieltjes transform on new generalized functions will parallel the construction of the Fourier transform developed by Colombeau [2, 3]. We will require some Fourier techniques in the Stieltjes transform development. Therefore, a very brief exposition is included on its construction. As is well known the Fourier transform of a rapid descent test function, $\phi(x) \in \mathscr{Y}\left(\mathbb{R}^{n}\right)$, is defined by the integral

$$
\hat{\phi}(w)=\int_{\mathbb{R}^{n}} e^{-i x w} \phi(x) d x,
$$

where $x \cdot w=x_{1} w_{1}+\cdots+x_{n} w_{n}$. The inverse Fourier transform is then defined by the integral

$$
\hat{\mathfrak{g}}^{-1}(x)=(2 \pi)^{-n} \int_{\mathbb{R}^{n}} e^{-i x w} \mathcal{g}(w) d w=(2 \pi)^{-n} \hat{\mathfrak{g}}(-x) .
$$

It is well known that these transforms give us a homeomorphism on the space, $\mathscr{Y}\left(\mathbb{R}^{n}\right)$.

We extend the Fourier transform to new generalized functions by applying the transform to the representatives belonging to the coset class of functions identified with 
the new generalized function, $G$. Again let $R$ be a member of the coset class belonging to the space, $\varepsilon_{m, \tau}\left(\mathbb{R}^{n}\right)$. Then for $\phi \in \mathscr{A}_{N}$ and $\varepsilon>0$, we define

$$
\hat{R}\left(\phi_{\varepsilon}, w\right)=\int_{\mathbb{R}^{n}} e^{-i x w} R\left(\phi_{\varepsilon}, x\right) \hat{\phi}(\varepsilon x) d x
$$

for all $w \in \mathbb{R}^{n}$.

Since $\left|e^{-i x w}\right|=1$, we immediately have $\hat{R} \in \varepsilon_{m, \tau}\left(\mathbb{R}^{n}\right)$ and its proof is completed in a similar manner as the existence of the integral immediately following Definition 3.1. Since the kernel of the Stieltjes transform is somewhat complicated as compared to the kernel component, $e^{-i x w}$, in the Fourier transform its application to new generalized functions becomes difficult.

We also note that if $f \in \mathscr{Y}\left(\mathbb{R}^{n}\right)$, then the extended Fourier transform

$$
\int_{\mathbb{R}^{n}} e^{-i x w} f(x) \hat{\phi}(\varepsilon x) d x
$$

coincides with the classical Fourier transform

$$
\int_{\mathbb{R}^{n}} e^{-i x w} f(x) d x .
$$

Again this is proved by considering the difference of the two Fourier transforms and noting the special behavior of $\hat{\phi}(\varepsilon x)$, namely,

$$
|\hat{\phi}(\varepsilon x)-1| \leq C \varepsilon^{q+1} .
$$

These details can be found in [2, 3].

5. Stieltjes transforms on new generalized functions. We now turn our focus to the Stieltjes transform. A text [6] presents the classical Stieltjes transform whereby the existence is proved whenever the function $\alpha(x)$ satisfies the "big $\theta$ " condition,

$$
\alpha(x)=\theta\left(x^{1-\delta}\right),
$$

for $\delta$ sufficiently small. We will implement this result in our present situation.

We first prove two technical lemmas.

LEMMA 5.1. If $\phi(x) \in \mathscr{Y}$, then $\phi(x)=\theta\left(x^{1-\delta}\right)$ for $\delta$ small and positive.

Proof. We show that $\lim _{x \rightarrow \infty}|\phi(x)| / x^{1-\delta} \leq C$.

Since $\phi(x) \in \mathscr{Y}$, we have

$$
|\phi(x)|<\frac{C}{\left(1+x^{2}\right)^{m}}=\frac{C}{\left(1+x^{2}\right)^{m}} \frac{x^{1-\delta}}{x^{1-\delta}}=\left[\frac{C}{x^{1-\delta}}\right]\left[\frac{x^{1-\delta}}{\left(1+x^{2}\right)^{m}}\right] .
$$

Let $m=1$ in (5.2) and then we have

$$
\lim _{x \rightarrow \infty}\left[\frac{C}{x^{1-\delta}}\right]\left[\frac{x^{1-\delta}}{\left(1+x^{2}\right)}\right]=\lim _{x \rightarrow \infty}\left[\frac{C}{x^{1-\delta}}\right] \lim _{x \rightarrow \infty}\left[\frac{1}{\left(x^{\delta-1}\right)+x^{1-\delta}}\right]=0 .
$$

Thus the result immediately follows. 
Now since $\phi(x) \in \mathscr{Y}$, then $\hat{\phi}(x)$ is also a member of $\mathscr{S}$ satisfying condition,

$$
|\hat{\phi}(x)| \leq \frac{C_{p}}{\left(1+|x|^{p}\right)}, \quad p \in \mathbb{N} .
$$

LEMMA 5.2. If $\phi(x) \in \mathscr{Y}$ and $R\left(\phi_{\varepsilon}, x\right) \in \varepsilon_{m, \tau}\left(\mathbb{R}^{n}\right)$, then $\hat{\phi}(\varepsilon x) \cdot R\left(\phi_{\varepsilon}, x\right)=\theta\left(x^{1-\delta}\right)$ for $\delta$ sufficiently small and positive.

Proof. Again we show that

$$
\lim _{x \rightarrow \infty} \frac{\left|\hat{\phi}(\varepsilon x) \cdot R\left(\phi_{\varepsilon}, x\right)\right|}{x^{1-\delta}} \leq C .
$$

By condition (2.7), it is clear that $R\left(\phi_{\varepsilon}, x\right)$ satisfies

$$
\left|R\left(\phi_{\varepsilon}, x\right)\right| \leq C \frac{\left(1+|x|^{N}\right)}{\varepsilon^{N}},
$$

and it can then be clearly shown [4] that $|\hat{\phi}(\varepsilon x)|<C_{p} / \varepsilon^{p}\left(1+|x|^{p}\right)$ for all $x$.

Then the product, $\hat{\phi}(\varepsilon x) \cdot R\left(\phi_{\varepsilon}, x\right)$ satisfies

$$
\begin{aligned}
\left|\hat{\phi}(\varepsilon x) R\left(\phi_{\varepsilon}, x\right)\right| & <\frac{C_{p}}{\varepsilon^{p}\left(1+|x|^{p}\right)} \cdot C \frac{\left(1+|x|^{N}\right)}{\varepsilon^{n}} \frac{x^{1-\delta}}{x^{1-\delta}} \\
& \leq \frac{k}{x^{1-\delta}}\left[\frac{1+|x|^{N+1}}{\varepsilon^{N+p}}\right]\left[\frac{1}{1+|x|^{p}}\right]
\end{aligned}
$$

and selecting $p>N+1$ proves the desired result.

We now define the Stieltjes transform of $G$ to be the following integral transform.

DEFINITION 5.3. For $R\left(\phi_{\varepsilon}, x\right) \in \varepsilon_{m, \tau}\left(\mathbb{R}^{n}\right)$ and being a representative for $G$ together with $\hat{\phi}(\varepsilon x)$, the Stieltjes transform of $G$ is defined by the integral transform formula

$$
G_{\text {Stieltjes }}(S)=\int_{0}^{\infty} \frac{d\left(R\left(\phi_{\varepsilon}, x\right) \cdot \hat{\phi}(\varepsilon x)\right)}{S+x} .
$$

Implementing the requirement in expression (5.1) this transform is well defined.

\section{REFERENCES}

[1] J.-F. Colombeau, Differential Calculus and Holomorphy. Real and complex analysis in locally convex spaces, North-Holland Mathematics Studies, vol. 64, North-Holland Publishing Co., New York, 1982. MR 84i:46005. Zbl 506.46001.

[2] _ New Generalized Functions and Multiplication of Distributions, North-Holland Mathematics Studies, vol. 84, North-Holland Publishing Co., New York, 1984. MR 86c:46042. Zbl 532.46019.

[3] _ Elementary Introduction to New Generalized Functions, North-Holland Mathematics Studies, vol. 113, North-Holland Publishing Co., New York, 1985. MR 87f:46064. Zbl 584.46024.

[4] J. Schmeelk, Some Wavelet Transforms in New Generalized Functions, International Conference of Recent Developments in Mathematical Analysis and Applications to Industrial Problems, Banaras Hindu University, Varanasi, India, preprint, March 2-5 1998. 
[5] L. Schwartz, Sur l'impossibilité de la multiplication des distributions, C. R. Acad. Sci. Paris 239 (1954), 847-848 (French). MR 16,265e. Zbl 056.10602.

[6] D. V. Widder, The Laplace Transform, Princeton Mathematical Series, vol. 6, Princeton University Press, New Jersey, 1941. MR 3,232d. Zbl 063.08245.

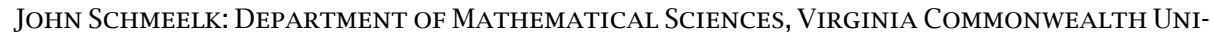
VERSITY, RICHMOND, VA 23284-2014, USA 


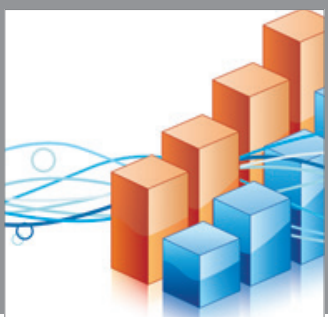

Advances in

Operations Research

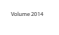

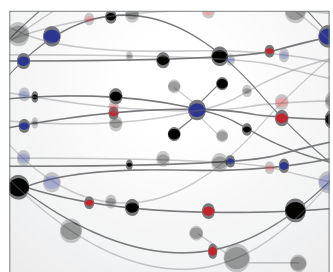

\section{The Scientific} World Journal
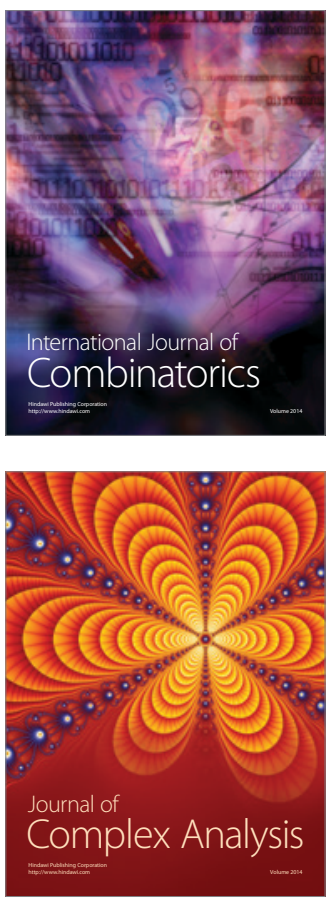

International Journal of

Mathematics and

Mathematical

Sciences
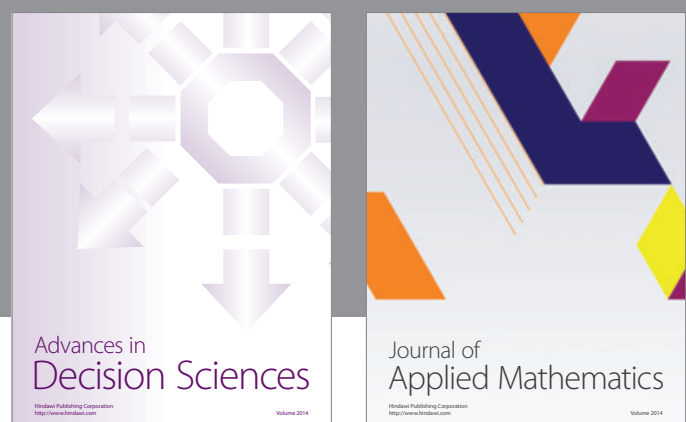

Journal of

Applied Mathematics
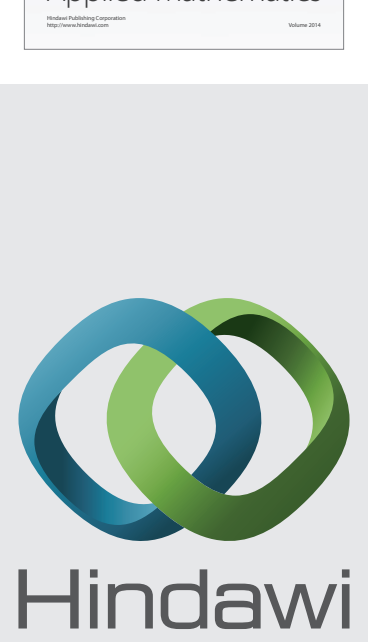

Submit your manuscripts at http://www.hindawi.com
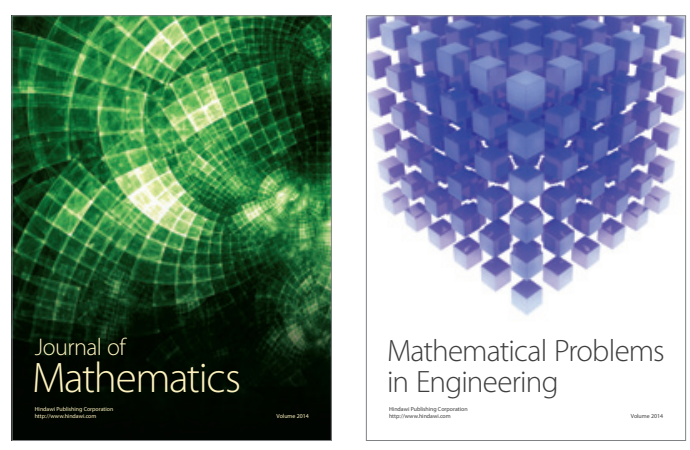

Mathematical Problems in Engineering
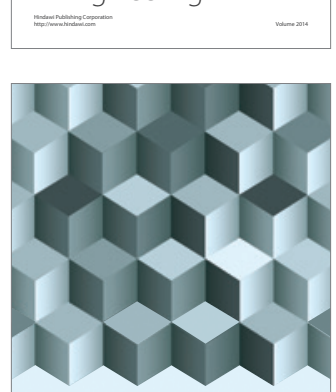

Journal of

Function Spaces
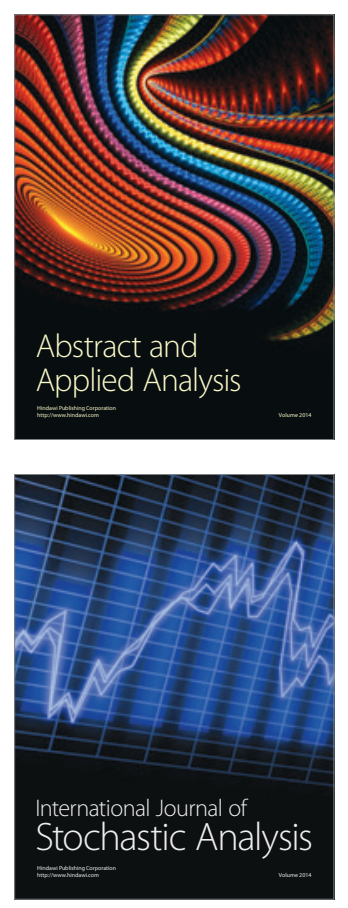

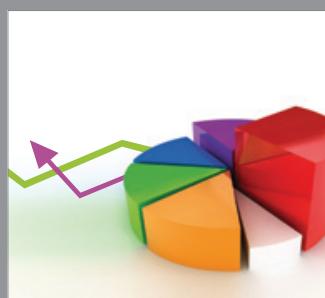

ournal of

Probability and Statistics

Promensencen
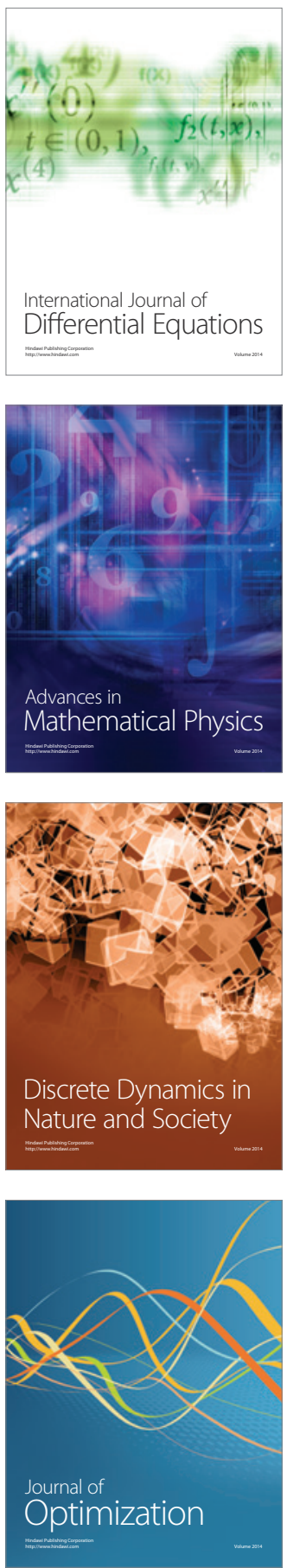